\title{
ESTIMACIÓN DE LA BIOMASA CON RELACIONES ALOMÉTRICAS EN DOS ESPECIES DE FRUTALES: THEOBROMA CACAO L. (CACAO) Y THEOBROMA GRANDIFLORUM (WILLD. EX SPRENG.) K.SCHUM. (COPOAZÚ)
}

\author{
Percy MARTÍNEZ ${ }^{1}$, Jorge SOLIGNAC ${ }^{2}$, Ricardo ZÁRATE ${ }^{1}$, Lizardo FACHÍN ${ }^{1}$, José T. MACO ${ }^{1}$ y Anita \\ Rocío JARAMA' \\ 1 Instituto de Investigaciones de la Amazonía Peruana. Programa de Investigación en Cambio Climático Desarrollo \\ Territorial y Ambiente (PROTERRA); Av. Quiñones km 2.5, San Juan Bautista, Maynas, Loreto, Perú. Correos \\ electrónicos: pmartinez@iiap.org.pe (P. Martínez); Ifachin@iiap.org.pe (L. Fachín); rzarate@iiap.org.pe (R. Zárate); \\ jmaco@iiap.org.pe (J. Maco); rjarama@iiap.org.pe. \\ 2. Gobierno Regional de Loreto. Programa de Manejo de Recursos Forestales y de Fauna Silvestre; Jr. Ricardo Palma 113. \\ Correo electrónico: soli217@hotmail.com (J. Solignac).
}

\section{RESUMEN}

Se elaboró un modelo alométrico para estimar la biomasa aérea total a partir de la variable altura en dos especies de frutales de dos años de edad: Theobroma cacao L. (cacao) y Theobroma grandiflorum (Willd. ex Spreng.) K.Schum. (copoazú). Estas especies se cultivan, asociadas con otras especies, en sistemas frutales de algunas áreas cercanas a la carretera Iquitos - Nauta.

El estudio utilizó un método destructivo con 18 individuos por especie. Se extrajeron submuestras del fuste, hojas, ramas y ramillas que fueron sometidas a un proceso de secado en hornos para obtener su peso seco y finalmente ordenar, tabular y analizar los datos para generar los modelos por especie. A partir de los resultados obtenidos de la biomasa total y por cada componente, se formaron modelos alométricos para cada una de las especies en función de la variable altura total. Las ecuaciones alométricas con el coeficiente de determinación $\left(\mathrm{R}^{2}\right)$ mayor a 0,80 fueron las cúbicas: $\mathrm{Y}=0,16+\left(-2,74 \mathrm{X}^{2}\right)+1,52 \mathrm{X}^{3}, \mathrm{y} \mathrm{Y}=0,62+4,04 \mathrm{X}$ $0,49 \mathrm{X}^{2}+2,062 \mathrm{X}^{3}$ para Theobroma cacao y Theobroma grandiflorum donde $\mathrm{Y}$ es la biomasa aérea $(\mathrm{t})$ y $\mathrm{X}$ es la altura total (m). En las dos ecuaciones el coeficiente de determinación $\left(R^{2}\right)$ fue mayor a 0,80 .

PALABRAS CLAVE: Biomasa, relaciones alométricas, Theobroma cacao, Theobroma grandiflorum, frutales amazónicos.

\section{BIOMASS ESTIMATE WITH ALLOMETRIC RELATIONSHIPS OF TWO FRUIT SPECIES NEAR TO IQUITOS, LORETO, PERU}

\section{ABSTRACT}

The aim of this study was to generate an allometric model to estimate total aboveground biomass from the variable height in two species of fruit of two years of age Theobroma cacao L. (Cacao) and Theobroma grandiflorum (Willd. ex Spreng.) K.Schum. (copoazú). These species are found close to Iquitos - Nauta highway in fruit systems associated with other species.

The study used a destructive method with 18 individuals for each species. Sub field samples were taken from stem, leaves, branches and twigs strata that were subjected to a drying process to obtain its dry weight and finally the data was sort, tabulate and analyze in order to generate models by species.

Allometric equations were obtained based on allometric variables in function of total biomass and total height, for each specie: Theobroma cacao $\mathrm{Y}=0,16+\left(-2,74 \mathrm{X}^{2}\right)+1,52 \mathrm{X}^{3}$, and Theobroma grandiflorum $\mathrm{Y}=$ $0,62+4,04 X-0,49 X^{2}+2,062 X^{3}$, where $Y$ is total biomass $(t), X$ is the height $(m)$. In both equations the coefficient of determination $\left(R^{2}\right)$ was more than 0,80 .

KEYWORDS: Biomass, Allometric Relationships, Theobroma cacao, Theobroma grandiflorum. 


\section{INTRODUCCIÓN}

El aumento de la concentración de $\mathrm{CO}_{2}$ en la atmósfera es una preocupación mundial. En los últimos 200 años el incremento fue de 280 a 350 ppm de $\mathrm{CO}_{2}$ en la atmósfera y el aumento continúa con un incremento promedio superior a $1 \mathrm{ppm}$ al año (Deans et al., 1996). Las plantas utilizan $\mathrm{CO}_{2}$ y liberan $\mathrm{O}_{2}$ durante el proceso de la fotosíntesis. A diferencia de las especies anuales, los árboles almacenan los fotoasimilados en componentes de carbono en sus estructuras leñosas por periodos prolongados, por lo que se les debe considerar como reservas naturales de carbono. La capacidad para almacenar carbono en forma de biomasa aérea varía en función de la composición florística, la edad y la densidad de población de cada estrato por comunidad vegetal (Schulze et al., 2000). La determinación de esta capacidad constituye un reto cuando se trata de evaluar el potencial de sistemas forestales, naturales, alterados o inducidos por el hombre.

En las últimas décadas, se ha prestado considerable atención a la estimación de biomasa de árboles individuales y rodales forestales. Se han desarrollado ecuaciones en relación a la biomasa o sus componentes (raíces, hojas, ramas y fuste) con las características dasométricas de los árboles. El interés radica en entender la productividad del sitio y en comparar la productividad con modelos convencionales de rendimiento e incremento (Pastor y Bockheim, 1981).

En la actualidad, los estudios sobre biomasa tienen como finalidad entender los ciclos de la energía y de los nutrientes. También se están usando para ver el efecto de la vegetación en el ciclo global del $\mathrm{CO}_{2}$ (Brown, 1997). Algunos modelos de $\mathrm{CO}_{2}$ (Mákelá, 1997; Mohren, 1994) incluyen la estimación de la biomasa o una función de la biomasa como el volumen, sus componentes o algunos parámetros relacionados, para establecer los flujos de este gas entre la vegetación (Schroeder et al., 1997; TerMikaelian y Korzukhin, 1997; Brown et al., 1989; Návar et al., 2001).

Es necesario mejorar la estimación de la biomasa aérea con una mayor precisión, con el propósito de modelar los flujos de carbono por cambio del uso de la tierra. Los resultados obtenidos dependerán en gran parte de las cuantificaciones de biomasa de los sistemas de frutales en la Amazonía.

Existe muy poca información sobre la estimación de biomasa en especies de frutales nativos. Es por esta razón que el objetivo de este trabajo fue estimar la biomasa aérea total en base a un modelo alométrico a partir de la variable altura total en dos especies, Theobroma cacao L. (cacao) y Theobroma grandiflorum (Willd. ex Spreng.) K.Schum. (copoazú), en sectores aledaños a la carretera Iquitos Nauta, Loreto-Perú.

\section{MATERIAL Y MÉTODOS}

\section{ÁREA DE ESTUDIO}

El estudio se desarrolló en las comunidades "24 de Octubre", "San Lucas" y "3 de Octubre" del distrito de San Juan Bautista, departamento de Loreto, Perú. Las coordenadas geográficas y mapa de ubicación se detallan en la figura 1 .

\section{A. SUELO}

Los suelos, de donde proceden las especies estudiadas, se ubican en terrazas medias onduladas arcillosas, con pendiente plana a ligeramente inclinada. Presentan drenaje bueno a moderado, permeabilidad moderadamente rápida, no se observa escurrimiento superficial. Químicamente son de reacción ultra ácida a extremadamente ácida $(\mathrm{pH}$ 2.26 a 4.35). Tienen alto porcentaje de materia orgánica y bajo fósforo en la capa superficial (Escobedo 2012).

\section{B. FISIOGRAFÍA}

El área de estudio presenta topografía plana a ligeramente ondulada, conformada por terrazas altas y medias con pendiente de 0 a $8 \%$. Incluye el paisaje de terrazas antiguas Pleistocénicas, (Escobedo y Torres 2012).

\section{CLIMA}

Climáticamente la zona se caracteriza por ser cálida, tropical y húmeda durante todo el año. $\mathrm{La}$ precipitación pluvial varía de 2,600 a 3,000 mm por año, distribuida de tal forma que no hay un período seco extenso, ni mes en el que la precipitación sea menor de $100 \mathrm{~mm}$. Las precipitaciones mayores ocurren en el mes de abril y las menores entre julio y agosto. En cuanto a las temperaturas, los meses más calurosos ocurren a finales del invierno, con mínimas medias de $20-23{ }^{\circ} \mathrm{C}$ y máximas entre $30-33$ ${ }^{\circ} \mathrm{C}$. Las medias anuales oscilan en torno a los $27^{\circ} \mathrm{C}$, y las máximas absolutas históricas alcanzan los 40 ${ }^{\circ} \mathrm{C}$. La variación diaria de la temperatura oscila entre 10 y $12^{\circ} \mathrm{C}$, lo que es mucho mayor que la variación anual, que apenas llega a ser de 1 a $2{ }^{\circ} \mathrm{C}$. (Paredes, 2012).

\section{ESPECIES DE FRUTALES}

\section{A.- ESPECIES ESTUDIADAS}

Se han considerado dos especies de frutales: Theobroma cacao L. (cacao) y Theobroma grandiflorum (Willd. ex Spreng.) K.Schum. (copoazú), debido a la importancia económica que ambos tienen para la región (González, 2007). 


\section{B.- SISTEMA DE CULTIVOS DE LOS FRUTALES}

El sistema o cultivo estába formado por cuatro o cinco especies de frutales nativos, como: Pouteria caimito (Ruiz \& Pav.) Radlk. (caimito), Theobroma bicolor Humb. \& Bonpl. (macambo), Rollinia mucosa (Jacq.) Baill. (anona), Persea americana Mill. (palta), Anacardium occidentale L. (casho), Theobroma cacao L. (cacao), Theobroma grandiflorum (Willd. ex Spreng.) K.Schum. (copoazú), y la superficie de cada sistema de frutales era de 0.5 ha, con una edad de dos años.

\section{TRATAMIENTO DE LOS INDIVIDUOS}

En función de la disponibilidad de los árboles de cada especie, se realizó la selección de manera aleatoria. Con el propósito de aumentar el intervalo de variación de los árboles medidos y dar validez a las ecuaciones de regresión, se juntaron un total de 36 individuos (18 individuos por especie), en clases de alturas, desde 0.60 a 2 metros de altura (desde la base del tallo hasta la copa).

Los árboles, después de ser derribados, fueron divididos en dos componentes: el tronco, que se seccionó en partes manejables para facilitar su pesaje, y la copa, que estaba conformada por hojas, ramas gruesas y ramas finas. Estas partes fueron pesadas en una balanza mecánica con una capacidad de $5 \mathrm{~kg}$, previamente instalada en el área de estudio. De esta manera se pudo obtener de forma separada el peso fresco de cada componente del árbol. Posteriormente, se tomaron al azar tres submuestras de $100 \mathrm{~g}$ del fuste, ramillas y hojas. Todas las submuestras fueron ordenadas, codificadas por individuo y enviadas al laboratorio para ser secadas al horno por 48 horas a $70{ }^{\circ} \mathrm{C}$ para obtener su peso seco constante, de acuerdo con la metodología empleada por Arreaga (2002). Se registró el peso seco de cada una de las muestras por componente. Mediante la diferencia entre el peso verde y el peso seco de las muestras se determinó el contenido de humedad. Este valor se descontó al peso verde de cada una de las secciones del fuste y de la copa, de esta forma se obtuvo la biomasa por componente estructural de todo el árbol.

\section{ANÁLISIS DE DATOS}

Obtenida la biomasa total y la altura total de cada individuo, se procedió a realizar el análisis de regresión entre la biomasa aérea (fuste+copa) como variable dependiente y la altura como variable independiente. En este trabajo se usaron los modelos matemáticos (Tabla 1) del software SPSS 20, así como la aplicación Excel para hojas de cálculo.

\section{RESULTADOS Y DISCUSIÓN}

A los dos años de edad, la altura total de las especies muestreadas en los sistemas de frutales nativos variaron de 0,9 a 1,97 $\mathrm{m}$ y la biomasa de 0,47 a 2,24 kg para la especie Theobroma cacao (cacao). Para Theobroma grandiflorum (copoazú) la altura varió de 0,62 a 2,07 m y la biomasa de 0,47 a 4,77 kg, (para mayores detalles ver la tabla 2).

Con el fin de evitar valores extremos y lograr desarrollar una ecuación consistente, se trabajó con los datos de 18 individuos por especie, encontrándose una distribución relativa variable de la biomasa total por cada individuo, resultado provocado por algunos factores físicos (nutrientes del suelo, drenaje, luminosidad, precipitación, temperatura, entre otros) y biológicos (factores externos como plagas, competencia de otras especies por el mismo hábitat y factores internos; como la genética de los individuos), así como también por las diferencias de manejo que algunos individuos recibieron, afectando este hecho al desarrollo de una biomasa normal (Moraes, 2001).

Se determinaron 11 ecuaciones de relación entre la biomasa y la altura total. En la especie Theobroma grandiflorum (copoazú), el coeficiente de determinación varió de 0,444 a 0,980 , con un promedio de 0,816 , aceptando a la ecuación cúbico con 0,980. En la especie Theobroma cacao (cacao), el coeficiente de determinación varió de 0,444 a 0,856 , con un promedio de 0,685 , aceptando a la ecuación con 0,856 . El coeficiente de correlación es más alto en Theobroma grandiflorum (copoazú) que en Theobroma cacao (cacao).

Los parámetros del modelo y la dispersión de puntos de los valores de biomasa observados se presentan en la figura 2 y 3 .

En la figura 2 se puede observar que la biomasa total aumenta con el incremento de altura y el diámetro del árbol. Sin embargo, los datos se muestran dispersos debido a que la biomasa del fuste incluye los factores altura y diámetro, los cuales causan variabilidad en el valor de la biomasa total.

En varios estudios se han registrado variaciones de los valores de biomasa en los componentes del árbol, ya que estos dependen tanto de los hábitos de crecimiento, como de las características ambientales del lugar donde se desarrollan (Garcidueñas, 1987). Otros factores que influyen en la variación de la biomasa son: características de la especie, tamaño del árbol (altura y diámetro), diámetro en la base de la copa y atributos del rodal (Gayoso et al., 2002).

Los resultados muestran que es factible encontrar una ecuación para la estimación de la biomasa de Theobroma cacao (cacao) y Theobroma grandiflorum (copoazú). La ecuación que presentó un mejor ajuste incluye como variable independiente a la altura total. 
La altura total fue incluida como variable en todos los modelos, debido a que es una variable fácil de medir en el campo. Al incluirla recogemos de manera indirecta información sobre el ambiente competitivo en el que se desarrolla un árbol, así como la edad del rodal.(Wirth et al, 2004).

La utilización de estas ecuaciones en la estimación de la biomasa total debe ser tomada con precaución cuando se realicen inventarios de biomasa en plantaciones de Theobroma cacao (cacao) y Theobroma grandiflorum (copoazú). Su aplicabilidad es más eficiente en el rango de alturas observadas en este estudio. Cuando aplicamos estas ecuaciones a individuos de mayor altura las estimaciones de biomasa podrían resultar sesgadas. Otra fuente de variación adicional que incluye el error asociado con la selección de las unidades muéstrales, son las consideraciones más importantes en el error asociado con la selección aleatoria del rodal (Parresol, 1999). Los gradientes en altitud sobre el nivel del mar, vinculados con otras variables asociadas con la pendiente, originan unidades de muestra diferentes en estructura y diversidad, pudiéndose incrementar considerablemente el error en la estimación de la biomasa total. La inclusión de nuevas variables en las ecuaciones de biomasa es materia de estudios adicionales para este sistema.

\section{AGRADECIMIENTOS}

Expresamos nuestro agradecimiento a Fidel Apuela Vásquez, Mario Escobedo Yaicate, Wilson Ríos Ríos, por permitirnos desarrollar el proyecto dentro de sus sistemas de cultivos de frutales. A la Facultad de Ciencias Forestales de la Universidad Nacional de la Amazonía peruana, por las facilidades proporcionadas en el uso de su laboratorio de Anatomía de la Madera. 


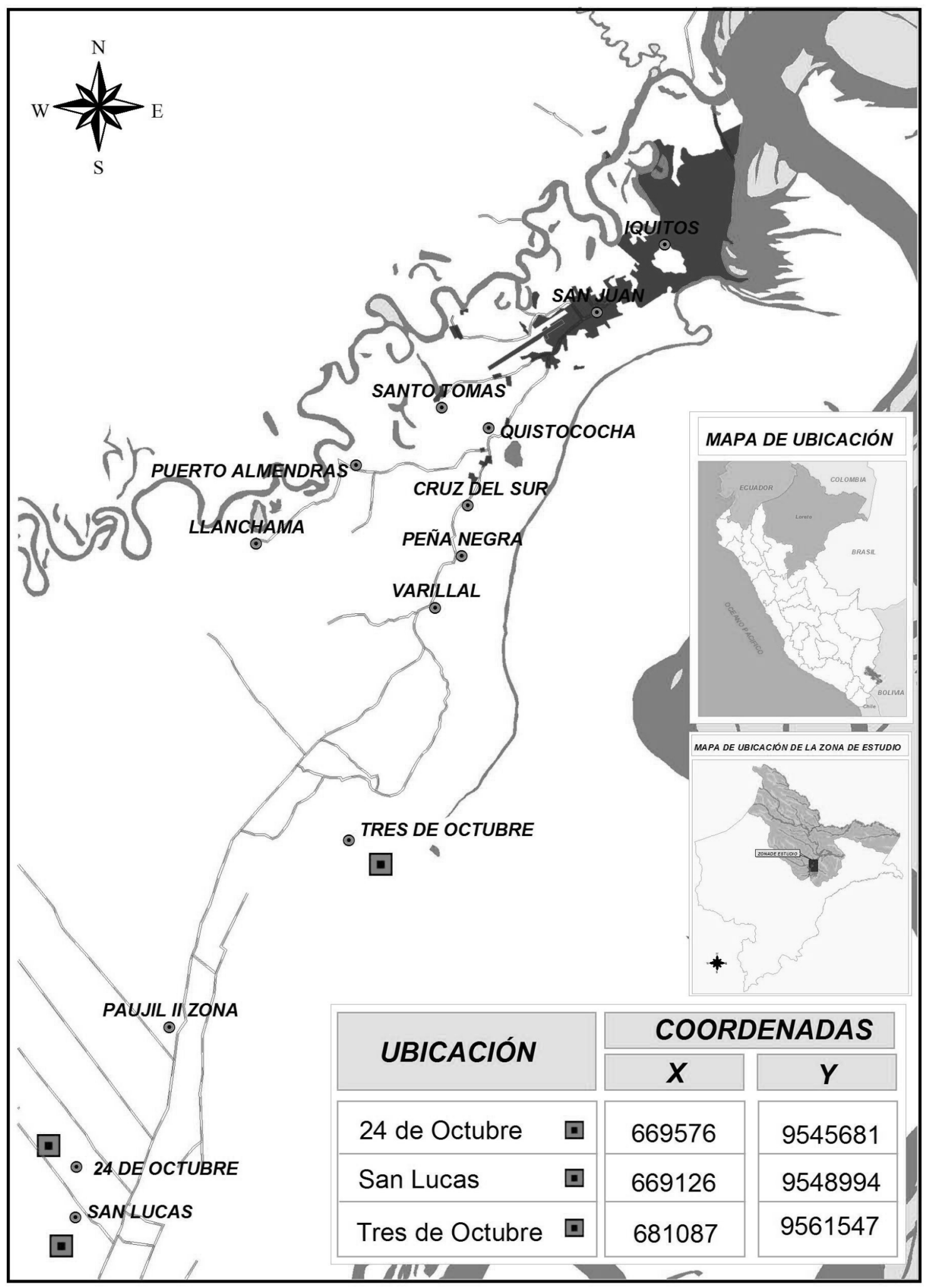

Figura 1. Mapa de ubicación de los sitemas frutales nativos 
FOLIA ESTIMACIÓN DE LA BIOMASA CON RELACIONES ALOMÉTRICAS EN DOS ESPECIES DE FRUTALES: THEOBROMA CACAO L. (CACAO) Y THEOBROMA GRANDIFLORUM (WILLD. EX SPRENG.) K.SCHUM. (COPOAZÚ)

Tabla 1. Modelos alométricos para la estimación de la biomasa por especie

\begin{tabular}{lll}
\hline$N^{\mathbf{N}}$ & $\begin{array}{c}\text { MODELO } \\
\text { MATEMÁTICO }\end{array}$ & \multicolumn{1}{c}{ ECUACIONES } \\
\hline 1 & Lineal & $\mathrm{Y}=\mathrm{b} 0+(\mathrm{b} 1 \times \mathrm{t})$ \\
2 & Logarítmica & $\mathrm{Y}=\mathrm{b} 0+(\mathrm{b} 1 \times \mathrm{Ln}(\mathrm{t}))$ \\
3 & Inversa & $\mathrm{Y}=\mathrm{b} 0+\left(\mathrm{b} 1 \mathrm{t}^{-1}\right)$ \\
\hline 4 & Cuadrática & $\mathrm{Y}=\mathrm{b} 0+(\mathrm{b} 1 \times \mathrm{t})+(\mathrm{b} 2 \times \mathrm{t} 2)$ \\
5 & Cúbica & $\mathrm{Y}=\mathrm{b} 0+(\mathrm{b} 1 \times \mathrm{t})+(\mathrm{b} 1 \times \mathrm{t} 2)+(\mathrm{b} 1 \times \mathrm{t} 3)$ \\
6 & Compuesta & $\mathrm{Y}=\mathrm{b} 0 \times\left(\mathrm{b} 1 \mathrm{t}^{-1}\right)$ \\
\hline 7 & Potencial & $\mathrm{Y}=\mathrm{b} 0 \times(\mathrm{t} \mathrm{b} 1)$ \\
8 & S & $\mathrm{Y}=\mathrm{e}\left(\mathrm{b} 0\left(\mathrm{~b} 1 \mathrm{t}^{-1}\right)\right.$ \\
9 & Crecimiento & $\mathrm{Y}=\mathrm{e}(\mathrm{b} 0(\mathrm{~b} 1 \times \mathrm{t})$ \\
10 & Exponencial & $\mathrm{Y}=\mathrm{b} 0(\mathrm{e}(\mathrm{b} 1 \times \mathrm{t})$ \\
11 & Logística & $\mathrm{Y}=1 /(1 \mathrm{u}-1+\mathrm{b} 0(\mathrm{~b} 1 \mathrm{t})$ \\
\hline
\end{tabular}

Tabla 2. Características dasométricos de Theobroma cacao (cacao), Theobroma grandiflorum (copoazú), muestreados

\begin{tabular}{lcccccc}
\hline $\mathbf{N}^{\mathbf{2}}$ & $\begin{array}{c}\text { ALTURA } \\
(\mathbf{m})\end{array}$ & $\begin{array}{c}\text { PESO VERDE } \\
(\mathbf{k g})\end{array}$ & $\begin{array}{c}\text { BIOMASA } \\
(\mathbf{k g})\end{array}$ & $\begin{array}{c}\text { ALTURA } \\
(\mathbf{m})\end{array}$ & $\begin{array}{c}\text { PESO VERDE } \\
(\mathbf{k g})\end{array}$ & $\begin{array}{c}\text { BIOMASA } \\
(\mathbf{k g})\end{array}$ \\
\hline 1 & 0.90 & 1.15 & 0.47 & 0.62 & 0.90 & 0.47 \\
\hline 2 & 0.92 & 0.88 & 0.32 & 1.12 & 1.10 & 0.58 \\
\hline 3 & 0.98 & 1.25 & 0.51 & 1.13 & 1.36 & 0.72 \\
\hline 4 & 1.04 & 1.00 & 0.40 & 1.18 & 1.28 & 0.67 \\
\hline 5 & 1.12 & 0.90 & 0.36 & 1.24 & 1.65 & 0.87 \\
\hline 6 & 1.24 & 1.15 & 0.47 & 1.26 & 1.90 & 1.00 \\
\hline 7 & 1.31 & 1.80 & 0.73 & 1.27 & 1.25 & 0.66 \\
\hline 8 & 1.32 & 1.30 & 0.53 & 1.30 & 1.80 & 0.95 \\
\hline 9 & 1.47 & 1.43 & 0.58 & 1.33 & 1.60 & 0.84 \\
\hline 10 & 1.50 & 1.50 & 0.61 & 1.47 & 2.20 & 1.16 \\
\hline 11 & 1.54 & 1.45 & 0.59 & 1.53 & 2.90 & 1.53 \\
\hline 12 & 1.62 & 1.50 & 0.61 & 1.60 & 2.87 & 1.52 \\
\hline 13 & 1.70 & 1.73 & 0.70 & 1.77 & 4.00 & 2.12 \\
\hline 14 & 1.78 & 2.81 & 1.14 & 1.82 & 5.64 & 2.98 \\
\hline 15 & 1.80 & 3.16 & 1.29 & 1.86 & 7.12 & 3.77 \\
\hline 16 & 1.86 & 6.26 & 2.56 & 1.98 & 8.00 & 4.24 \\
\hline 17 & 1.93 & 6.50 & 2.65 & 2.03 & 8.70 & 4.61 \\
\hline 18 & 1.97 & 5.50 & 2.24 & 2.07 & 9.00 & 4.77 \\
\hline
\end{tabular}


Tabla 3. Ecuaciones con sus $\mathrm{R}^{2}$ para Theobroma cacao (cacao)

\begin{tabular}{|c|c|c|c|c|c|c|}
\hline \multirow{2}{*}{ Ecuación } & \multicolumn{2}{|c|}{ Resumen del modelo } & \multicolumn{4}{|c|}{ Estimaciones de los parámetros } \\
\hline & R cuadrado & $\mathbf{F}$ & Constante & b1 & b2 & b3 \\
\hline Lineal & 0.599 & 23,916 & -1.446 & 1.645 & & \\
\hline Logarítmica & 0.521 & 17.418 & 0.224 & 2.100 & & \\
\hline Inversa & 0.444 & 12.765 & 2.796 & -2.527 & & \\
\hline Cuadrático & 0.836 & 38.251 & 5.363 & -8.510 & 3.562 & \\
\hline Cúbico & 0.856 & 44.609 & 1.685 & 0.000 & -2.745 & 1.502 \\
\hline Compuesto & 0.750 & 47.990 & 0.073 & 4.928 & & \\
\hline Potencia & 0.680 & 34.056 & 0.365 & 2.079 & & \\
\hline$S$ & 0.604 & 24.444 & 1.579 & -2.555 & & \\
\hline Crecimiento & 0.750 & 47.990 & -2.611 & 1.595 & & \\
\hline Exponencial & 0.750 & 47.990 & 0.073 & 1.595 & & \\
\hline Logística & 0.750 & 47.990 & 13.607 & 0.203 & & \\
\hline
\end{tabular}

\begin{tabular}{|c|c|c|c|c|c|c|}
\hline \multirow{2}{*}{ Ecuación } & \multicolumn{2}{|c|}{ Resumen del modelo } & \multicolumn{4}{|c|}{ Estimaciones de los parámetros } \\
\hline & R cuadrado & $\mathbf{F}$ & Constante & b1 & b2 & b3 \\
\hline Lineal & 0.814 & 70.023 & -3.333 & 3.516 & & \\
\hline Logarítmica & 0.651 & 29.833 & 0.400 & 4.138 & & \\
\hline Inversa & 0.444 & 12.800 & 4.712 & -3.880 & & \\
\hline Cuadrático & 0.972 & 257.920 & 3.221 & -6.206 & 3.360 & \\
\hline Cúbico & 0.980 & 224.891 & -0.625 & 4.041 & -4.909 & 2.062 \\
\hline Compuesto & 0.926 & 200.262 & 0.081 & 6.853 & & \\
\hline Potencia & 0.804 & 65.477 & 0.604 & 2.360 & & \\
\hline S & 0.607 & 24.746 & 2.039 & -2.328 & & \\
\hline Crecimiento & 0.926 & 200.262 & -2.515 & 1.925 & & \\
\hline Exponencial & 0.926 & 200.262 & 0.081 & 1.925 & & \\
\hline Logística & 0.926 & 200.262 & 12.365 & 0.146 & & \\
\hline
\end{tabular}


FOLIA

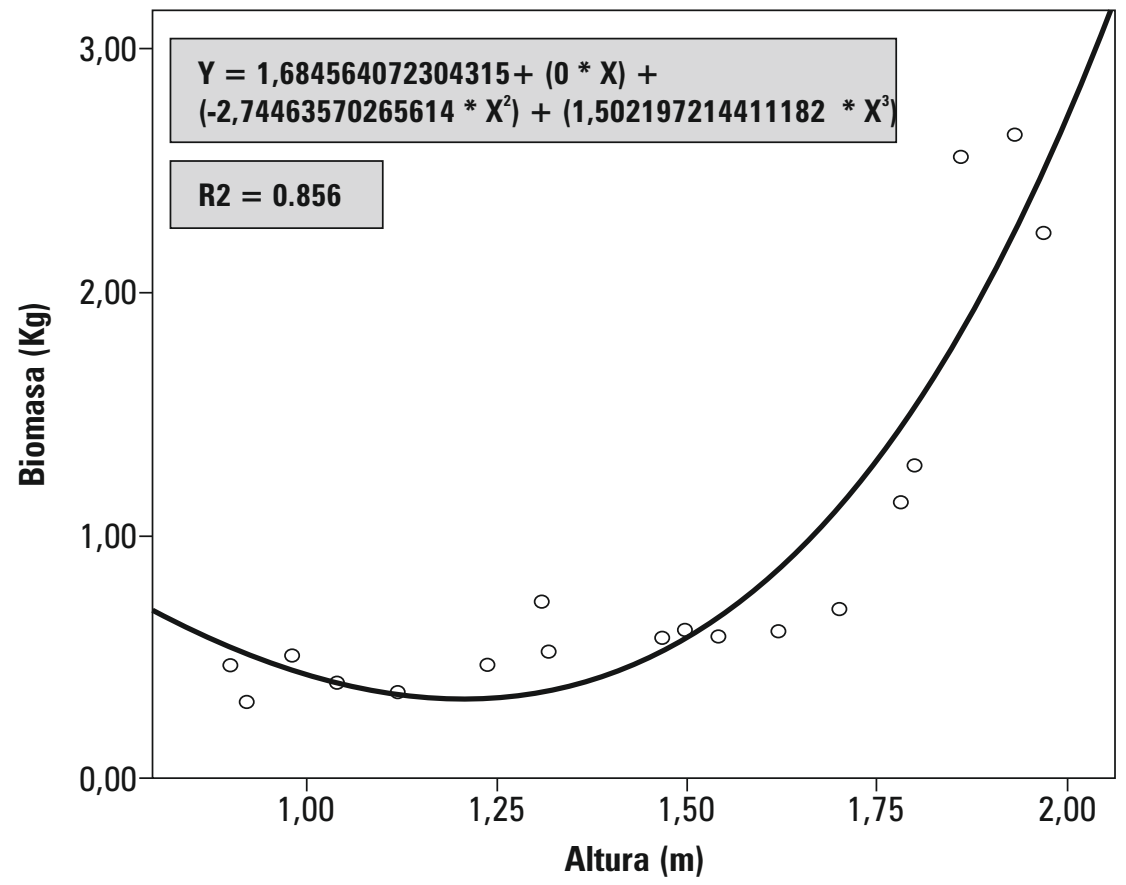

Figura 2. Diagrama de dispersión de los valores observados para estimar la biomasa en Theobroma cacao (cacao) y la línea de regresión generada con el modelo de ecuación cúbica.

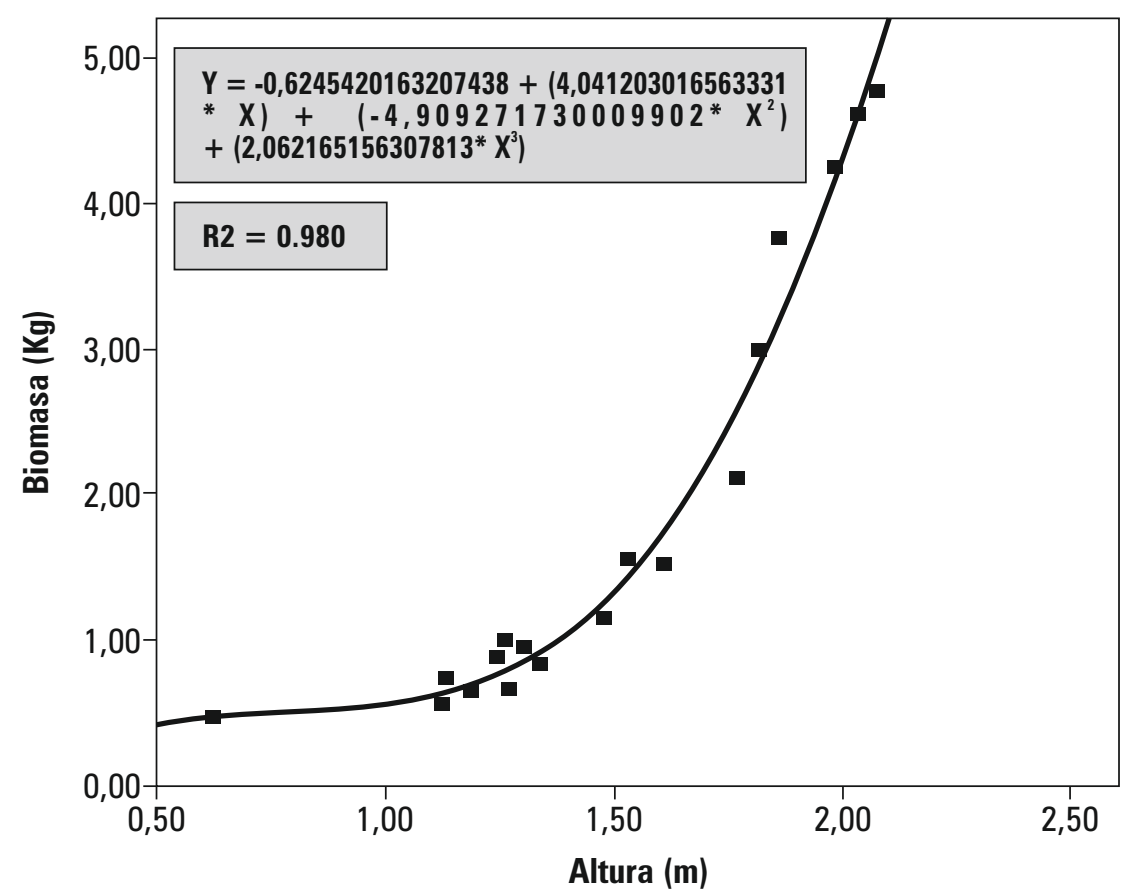

Figura 3. Dispersión de los valores observados para estimar la biomasa en Theobroma grandiflorum (copoazú) y la línea de regresión generada con el modelo cúbica. 


\section{BIBLIOGRAFÍA CITADA}

ARREAGA. W. 2002. Almacenamiento del carbono en bosques con manejo forestal sostenible en la reserva de Biosfera Maya, Peten, Guatemala. Tesis Mag. Sc. Turrialba, CR, CATIE. 111p.

BROWN, S. 1997. Los bosques y el cambio climático: el papel de los terrenos forestales como sumideros de carbono. In actas del XI Congreso Mundial Forestal: Recursos Forestales y Árboles. Vol 1. Antalya Turkia 1322 October of 1997.

CLUTTER J., J. FORSTON, L. PIENAAR, G. BRISTER, R. BAILEY, 1983. Timber Management. A quantitative approach. Krieger Publishing Company. Malabar, Florida.

DEANS, J. D., J. Mora., and J. Grace. 1996. Biomass relationships for tree species in regenerating semi-deciduous tropical moist forest in Cameeroon. For. Ecol. Manag. 88: 215-225.

ESCOBEDO, R. 2012. Suelos y capacidad de uso mayor de las tierras, documento temático. Proyecto Microzonificación Ecológica y Económica del Área de Influencia de la Carretera Iquitos-Nauta, convenio entre el IIAP y DEVIDA. Iquitos - Perú. Todos los derechos reservados. Queda prohibido reproducir

ESCOBEDO, R. TORRES, G. 2012. Fisiografía, documento temático. Proyecto Microzonificación Ecológica y Económica del Área de Influencia de la Carretera IquitosNauta, convenio entre el IIAP y DEVIDA. Iquitos - Perú

GARCIDUEÑAS, M. A. R. 1987. Producción de biomasa y acumulación de nutrientes en un rodal de Pinus montezumae Lamb. Tesis de Maestría. Colegio de Postgraduados. Montecillo, México. $243 \mathrm{p}$.

GAYOSO, J.; GUERRA, J. y ALARCON, D. 2002. Contenido de carbono y funciones de biomasa en especies nativas y exóticas. Valdivia (Chile): Universidad Austral de Chile, Proyecto medición de la capacidad de captura de carbono en bosques de Chile y promoción en el mercado mundial. Informe Final, Documento $\mathrm{N}^{\mathrm{o}} 1.53 \mathrm{p}$.

GONZALES, A. 2007. Frutales nativos amazónicos. Patrimonio alimenticio de la humanidad. IIAP. $76 \mathrm{p}$.

HUXLEY, J. S. 1932. Problems of Relative Growth. The Dial Press, New York. 276 p.

LOCATELLI, B., Y S. LEONARD 2001. Un método para medir el carbono almacenado en los bosques de Malleco (Chile). Bois et Forêts des Tropiques. 276(1): 69-80.

MÁKELÁ, A. 1997. A carbon balance model of growth and selfpruning in tree based on structural relationships. For. Sci. 43: 7-24.

MOHREN, F. 1994. CO2 Fix model. Institute of Forestry and Nature Research. Wageningen, Netherlands.

MORAES, C. 2001. Almacenamiento de carbono en bosques secundarios en el municipio de San Carlos, Nicaragua. Escuela de postgrado, Centro Agronómico de Investigación y Enseñanza, CATIE, Turrialba, Costa Rica.

NÁVAR C., J. J., N. GONZÁleZ B., Y J. GRACIANO L. 2001. Ecuaciones para estimar componentes de biomasa en plantaciones forestales de Durango, México. In: Simposio internacional medición y monitoreo de la captura de carbono en ecosistemas forestales. Valdivia, Chile. $12 \mathrm{p}$.

PAREDES, M. 2012. Clima, documento temático. Proyecto Microzonificación Ecológica y Económica del Área de Influencia de la Carretera Iquitos-Nauta, convenio entre el IIAP y DEVIDA. Iquitos - Perú

PARRESOL, B. 1999. Assessing tree and stand biomass: a review with examples and critical comparisons. For. Sci 45: 573-593.

PASTOR, J. AND J. G. BOCKHEIM. 1981. Biomass and production of an aspen mixed harwood-spodosol ecosystem in northern Wisconsin. Can. J. for. Res. 11: 132-138.

SCHROEDER, P., S. BROW, J. MO, R. BIRSSEY, AND C. CIESZEWSKI. 1997. Biomass estimation for temperate broadleaf forest of the United States using inventory data. For. Sci. 43 (3) $424-434$

SCHULZE, E. D., Ch. Wirth and M. Heimann. 2000. Managing forests after Kyoto. Science 289 (5487):2058-2059.

TER-MIKAELIAN, M. T. AND M. D. KORZUKHIN. 1997. Biomass equations for sixty five North American tree species. Forest Ecology and Management 97:1-24.

WIRTH, C., SCHUMECHER J., S. E. D. 2004. Generic biomass functions for Norway spruce in Central Europe a meta-analysis approach toward prediction and uncertainty estimation. Tree Physiology, 24, 121-139. 
